\title{
Les pères du théâtre médiéval. Examen critique de la constitution d'un savoir académique, sous la direction de Marie Bouhailk-gironès, Véronique Dominguez et Jelle Koopmans
}

\section{Paola Cifarelli}

\section{OpenEdition}

\section{Journals}

Édition électronique

URL : https://journals.openedition.org/studifrancesi/4615

DOI : $10.4000 /$ studifrancesi.4615

ISSN : 2421-5856

Éditeur

Rosenberg \& Sellier

Édition imprimée

Date de publication : 1 avril 2012

Pagination : 127-128

ISSN : 0039-2944

Référence électronique

Paola Cifarelli, "Les pères du théâtre médiéval. Examen critique de la constitution d'un savoir académique, sous la direction de Marie Bouhaïk-gironès, Véronique Dominguez et Jelle Koopmans », Studi Francesi [En ligne], 166 (I | LVI) | 2012, mis en ligne le 30 novembre 2015, consulté le 19 novembre 2021. URL: http://journals.openedition.org/studifrancesi/4615; DOI : https://doi.org/10.4000/studifrancesi.4615

Ce document a été généré automatiquement le 19 novembre 2021.

Studi Francesi è distribuita con Licenza Creative Commons Attribuzione - Non commerciale - Non opere derivate 4.0 Internazionale. 


\title{
Les pères du théâtre médiéval. Examen critique de la constitution d'un savoir académique, sous la direction de Marie Bouhaïk-gironès, Véronique Dominguez et Jelle Koopmans
}

\author{
Paola Cifarelli
}

\section{RÉFÉRENCE}

Les pères du théâtre médiéval. Examen critique de la constitution d'un savoir académique, sous la direction de Marie BOUHAÏK-GIRONÈs, Véronique DOMINGUEZ et Jelle KOOPMANS, Rennes, Presses Universitaires, 2010, pp. 281.

1 Ce volume rassemble les communications présentées lors du colloque international d'Amsterdam qui a eu lieu les 22 et 23 mars 2007 autour de questions d'ordre historiographique et épistémologique concernant le théâtre médiéval. En effet les articles, organisés en cinq sections, jettent tous un regard rétrospectif sur les textes fondateurs de la critique concernant le théâtre médiéval français. Dans l'introduction, M.B.-G. et V.D. tracent le cadre de référence et décrivent les enjeux de ce débat, qui s'articule autour de quelques points forts: l'étendue du champ d'études sur le théâtre médiéval, les rapports de celui-ci avec la littérature, l'aspect performatif et l'oralité en relation avec la transmission par les textes, les questions méthodologiques.

Dans la première partie («Les grands-pères: généalogie d'une pseudo-science»), l'essai de Robert CLARK (Les frères Parfaict et François de Beauchamp, historiens du théâtre médiéval au XVII siècle, pp. 23-34) a pour objet les véritables pionniers des études sur le théâtre médiéval que furent les frères François et Claude Parfaict, ainsi que leur contemporain François de Beauchamp; l'A. montre toute la richesse de la documentation qu'ils 
fournissent et l'intérêt de leurs recherches, malgré l'oubli où elles sombrent aujourd'hui. Denis HÜE suit par contre le sort d'un des textes dramatiques les plus célèbres en retraçant l'histoire du Pathelin entre l'époque de sa diffusion par le manuscrit et la réapparition, au 1853, du texte original; l'A. prend en considération les citations, les remaniements et les adaptations qui ont été élaborés au fil des siècles (Pour une histoire du "Pathelin": l'héritage des grands-pères, pp. 35-51). Le rôle joué par les érudits au sein des sociétés savantes au XVIII ${ }^{e}$ siècle est au cœur du chapitre rédigé par Katell LEVANT (Les sociétés savantes du Nord et leurs études sur le théâtre médiéval, pp. 53-70), où sont analysés les enjeux méthodologiques, les principes idéologiques et les résultats des recherches publiées au sein de la Société des Antiquaires de la Morinie, souvent essentielles pour la connaissance de l'activité théâtrale dans les provinces du Nord.

3 L'un des phares de la médiévistique française qui pourtant s'est peu occupé du théâtre est Joseph Bédier, à qui est consacré le chapitre IV, première section de la deuxième partie («Visions et dogmes»); Alain CORBELLARI (De Francisque Michel à Bédier: le problème du théâtre profane, pp. 73-84) analyse la seule contribution de l'illustre philologue dans le domaine dramatique en mettant en perspective les idées contenues dans l'essai sur les Commencements du théâtre comique en France (1890) et en montrant que cet article reflète l'évolution méthodologique subie par les études médiévales au tournant du siècle. Tout aussi marginale dans les études sur le théâtre est, à première vue, la place de Gaston Paris, qui procura l'édition critique de deux textes du théâtre religieux et n'écrivit qu'un nombre limité d'articles sur le sujet; toutefois, l'analyse des ouvrages de synthèse dus à la plume du célèbre savant permet à Ursula BÄHLER de dégager les lignes de force de sa réflexion sur le théâtre, conditionnée par ses principes idéologiques (Gaston Paris face au théâtre: si proche, si loin, pp. 85-99). Le chapitre qui achève cette deuxième section est consacré à Louis Petit de Julleville (Élisabeth LALou, Le dogme Petit de Julleville, pp. 101-118): sa personnalité, sa carrière, mais surtout sa méthode de travail et les idées qui sous-tendent ses recherches - enregistrées dans la bibliographie de ses œuvres placée à la fin de ce chapitre - permettent de tracer le portrait scientifique et humain du savant qui, plus que tout autre peut-être, mérite le titre de père des études sur le théâtre français du Moyen Âge.

4 La troisième partie de ce volume, intitulée «La création des genres et la constitution des corpus: sotties, farces et moralités», s'ouvre sur le chapitre consacré à Émile Picot, lui aussi «l'un des plus grands historiens du théâtre médiéval français» (p. 121). L'étude que Marie BOUHAїK-GIRONÈs consacre au monumental recueil rassemblé par l'éminent bibliographe (Le "Recueil Général des Sotties" d'Émile Picot ou la construction d'un genre dramatique, pp. 121-137) décrit ce véritable corps de doctrine en montrant l'effort de construction et de systématisation qui est à sa base, mais affirme également la nécessité de sortir des classements par genres que les recherches ultérieures ont prouvé être parfois inopérants: l'exemple de la Sottie de l'Astrologue montre qu'une approche nouvelle, fondée sur l'histoire des pratiques et l'étude des archives, est désormais indispensable. Ce chapitre fournit également une description très utile du 'fichier Picot' conservé à la Bibliothèque nationale de France, qui incitera peut-être les chercheurs à exploiter plus systématiquement cette véritable mine d'informations inédites. C'est encore un genre qui nécessite d'une remise en question à faire l'objet du chapitre Terra incognita: la longue 'invention' de la moralité française (XVI- $\mathrm{XXI}$ e siècles, pp.139-156) par Estelle DOUDET; l'A. examine les raisons qui ont rendu difficile l'étude systématique de la moralité, malgré les nombreuses éditions de textes procurées au cours des dernières 
décennies; elle retrace ensuite l'histoire de la critique autour de ce genre, qui suscite de nos jours un renouveau d'intérêt. Le chapitre suivant, consacré à la farce, est dû au grand spécialiste Jelle KOоPмANs ('Recueil de farces', histoire d'une notion et d'une pratique du XIX $X^{e}$ siècle à Gustave Cohen, pp.157-177), qui passe en revue les différents recueils rassemblés au fil des années, du manuscrit La Vallière édité entre 1831 et 1838 par Le Roux de Lincy et Michel au Recueil de farces inédites du Xv' siècle connu comme 'Recueil Cohen', en passant par le Recueil Trepperel publié par Eugénie Droz et Halina Lewika et par les différents projets d'édition annoncés qui n'ont pas abouti. Dans la dernière partie de ce chapitre, l'A. met en question la paternité de l'édition du 'Recueil Cohen' et annonce les grands projets éditoriaux actuellement en chantier.

La quatrième partie ("Questions de genre, questions de forme») est constituée de trois chapitres qui abordent des questions plus spécifiques, sans pour autant abandonner le point de vue rétrospectif qui caractérise cet ouvrage. Xavier LEROUX (La nature du texte dramatique à l'épreuve de la numérotation des vers, pp. 181-199) se penche sur un problème seulement à première vue marginal, à savoir la numérotation des vers lors de l'établissement du texte en vue d'une édition critique; en mettant en parallèle la pratique des grands éditeurs du passé, l'A. montre à quel point les choix dans ce domaine mettent en cause les questions concernant la définition du genre dramatique. Mario LONGTIN (La parole doublant l'action: maladresse ou choix esthétique? La critique et ses présupposés, pp. 201-211) montre que les conceptions esthétiques modelées sur les codes modernes peuvent nuire à l'appréciation du théâtre médiéval et fausser le jugement, comme c'est le cas pour les vers 'narratifs' qui glosent l'action dans le Mystère de saint Christofle: l'éditeur moderne émet un jugement sévère à ce propos, en utilisant des catégorisations qui ne tiendraient pas suffisamment compte du dispositif scénique de l'époque et du type de jeu représenté. Véronique DOMinguez ("Aucassin et Nicolette": une pièce de théâtre médiéval?, pp. 213-229) analyse un texte atypique en s'interrogeant sur la réelle possibilité que ce texte ait été composé pour la performance; après avoir retracé l'histoire critique de cette 'chantefable' et avoir étudié le texte dans son contexte manuscrit, l'A. met en question son appartenance au genre théâtral.

La dernière partie du volume aborde la question de la mise en scène du théâtre médiéval aux temps modernes, en prenant comme objet d'étude les représentations du groupe des Théophiliens, qui a porté sur scène trois spectacles entre 1933 et 1935; Isabelle RAGNARD (Influences musicologiques dans les premières représentations des Théophiliens (1933-1935), pp. 233-253) reconstruit les représentations du groupe animé par Gustave Cohen en examinant surtout la collaboration avec les musicologues André Pirro, professeur d'Histoire de la musique à la Sorbonne, Yvonne Rokseth, élève de celui-ci et Jacques Chailley, jeune président, directeur musical et metteur en scène du Groupe de Théâtre médiéval de la Sorbonne. C'est encore la troupe des Théophiliens et ses spectacles inspirés du théâtre médiéval qui font l'objet du chapitre dû à la plume d'Helen SOLTERER (Jouer le moyen âge: Gustave Cohen et la troupe théophilienne, pp. 255-281). 